2013

\title{
Adherence to Behavioral Targets and Treatment Attendance During a Pediatric Weight Control Trial
}

Kelly R. Theim

Denise E. Wilfley

Meghan M. Sinton mmsinton@wm.edu

Meghan M. Sinton

William \& Mary, mmsinton@wm.edu

\section{Recommended Citation}

Theim, K. R., Sinton, M. M., Goldschmidt, A. B., Van Buren, D. J., Doyle, A. C., Saelens, B. E., ... \& Wilfley, D. E. (2013). Adherence to behavioral targets and treatment attendance during a pediatric weight control trial. Obesity, 21(2), 394-397.

This Article is brought to you for free and open access by the Arts and Sciences at W\&M ScholarWorks. It has been accepted for inclusion in Arts \& Sciences Articles by an authorized administrator of W\&M ScholarWorks. For more information, please contact scholarworks@wm.edu. 


\title{
Adherence to Behavioral Targets and Treatment Attendance During a Pediatric Weight Control Trial
}

\author{
Kelly R. Theim ${ }^{1}$, Meghan M. Sinton ${ }^{2,3}$, Andrea B. Goldschmidt ${ }^{4}$,Dorothy J. Van Buren ${ }^{2}$, Angela C. Doyle ${ }^{5,6}$, \\ Brian E. Saelens ${ }^{7}$, Richard I. Stein ${ }^{8}$, Leonard H. Epstein ${ }^{9}$ and Denise E. Wilfley ${ }^{1,2}$
}

Objective: Better weight loss outcomes are achieved in adults and youth who adhere to obesity treatment regimens (i.e., session attendance and prescribed changes in weight control behaviors). However, more research is needed regarding children's adherence to a range of behaviors relevant for weight maintenance over long-term follow-up.

Design and Methods: Overweight children ( $N=101$, aged 7-12 years), along with an overweight parent, participated in a 20-week family-based behavioral weight loss treatment (FBT) and were then assigned to either a behaviorally focused or socially focused 16-week weight maintenance treatment (MT). Treatment attendance and child and parent adherence (i.e., reported use of skills targeted within treatment) were examined in relation to child percent overweight change from baseline to post-FBT, post-MT, and 2-year follow-up.

Results: Higher attendance predicted better child weight outcomes at post-MT, but not at 2-year followup. Adherence to self-regulatory skills/goal-setting skills predicted child weight outcomes at 2-year follow-up among the behaviorally focused MT group.

Conclusions: Future research is needed to examine mediators of change within family-based weight control interventions, including behavioral and socially based targets. Incorporating self-regulatory weight maintenance skills into a comprehensive MT may maximize children's sustained weight control.

Obesity (2013) 21, 394-397. doi:10.1038/oby.2012.94

The prevalence of pediatric overweight has increased dramatically (1). Family-based behavioral weight control programs lead to significant weight loss in children $(2,3)$, yet there is variability in response (4). Moreover, high attrition and poor adherence are common problems that may limit existing weight control treatments' efficacy (5).

In pediatric weight loss studies, attendance $(3,6)$ as well as adherence to targeted behaviors such as self-weighing and self-monitoring is associated with better weight loss outcomes $(7,8)$. Parental involvement is also associated with child success, perhaps via promoting children's behavioral adherence $(9,10)$. However, many pediatric studies of self-reported adherence focus only on short-term outcomes $(9,10)$. Further research is needed to extend these findings to include a detailed examination of which specific targeted behaviors (e.g., behavioral, social support-focused) are most associated with long-term outcome.

In this study, two novel family-based weight maintenance treatments (MTs) that used either a behaviorally based or socially based approach were examined. The primary outcome study (2) previously reported that children achieved better weight outcomes in MT as compared to a no-MT-control, with socially based treatment demonstrating the best efficacy, especially among children low in social problems. The present analyses sought to replicate previous findings regarding weight loss treatment attendance and adherence, and augment literature regarding whether treatment attendance and adherence to specific targeted behaviors within MT predicted children's short- and long-term weight outcomes.

\section{Methods and Procedures}

\section{Participants and procedure}

Participants were 101 overweight children (aged 7-12, $M=9.9 \pm$ 1.4 years; $71 \%$ girls), each with at least one overweight parent, involved in a randomized controlled trial of a 20 -week family-based

\footnotetext{
${ }^{1}$ Department of Psychology, Washington University, St. Louis, Missouri, USA ${ }^{2}$ Department of Psychiatry, Washington University School of Medicine, St. Louis, Missouri, USA ${ }^{3}$ Department of Psychology, The College of William and Mary, Williamsburg, Virginia, USA ${ }^{4}$ Department of Psychiatry and Behavioral Neuroscience, The University of Chicago, Chicago, Illinois ${ }^{5}$ Eating and Weight Disorders Center of Seattle, Seattle, Washington, USA ${ }^{6}$ Department of Psychology, University of Washington, Seattle, Washington, USA ${ }^{7}$ Department of Pediatrics, Seattle Children's Research Institute and University of Washington, Seattle, Washington, USA ${ }^{8}$ Department of Internal Medicine, Washington University School of Medicine, St. Louis, Missouri, USA ${ }^{9}$ Department of Pediatrics, State University of New York at Buffalo School of Medicine, Buffalo, New York, USA. Correspondence: Denise E. Wilfley (wilfleyd@psychiatry.wustl.edu)
}

Disclosure: The authors declared no conflict of interest.

See the online ICMJE Conflict of Interest Forms for this article.

Received: 25 July 2011 Accepted: 2 April 2012 First published online by Nature Publishing Group on behalf of The Obesity Society 3 May 2012. doi:10.1038/oby.2012.94 
behavioral weight loss treatment (FBT) (11) followed by randomization to one of two 16-week MTs. Families were permitted to advance to the MT phase regardless of weight loss or attendance/adherence during FBT. Families who completed FBT but were randomized to the no-MT-control $(n=49)$ were excluded from the present analyses. At least one parent/guardian attended with the child; all except one participating parent were overweight $(M$ BMI $=35.1 \pm 5.9 \mathrm{~kg} / \mathrm{m}^{2}$ ). At the start of the program, families were asked to pay an incentive deposit of $\$ 100$, which was returned to them in $\$ 25$ increments upon completion of each follow-up assessment (post-FBT, post-MT, 1-year follow-up, and 2-year follow-up). Telephone contacts and mailings were used to encourage families to return for distal follow-up assessments.

FBT taught families to modify their diet and physical activity through using behavioral weight loss skills (e.g., self-monitoring, stimulus control). Families were assigned to either social facilitation MT (SFM; $n=50$ ) or behavioral skills MT (BSM; $n=51$ ), both of which consisted of 16 weekly sessions, including individual family meetings and separate parent/child group meetings. MT sessions focused in part on continued dietary and physical activity recommendations to support weight maintenance (i.e., remaining within \pm $1.5 \mathrm{lbs}$ of post-FBT weight). However, the treatments differed in content. BSM built on FBT's behavioral approach and added weight maintenance-specific skills (e.g., "getting back on track" after a lapse and other weight maintenance skills training) and motivational enhancement techniques. SFM emphasized developing and utilizing social networks to support weight maintenance and decreasing social barriers to physical activity. For example, SFM included a focus on augmenting coping skills for eating- and weight-related teasing/criticism, as well as on promoting a positive body image. Self-monitoring in SFM included tracking peer-related activities involving healthy eating and physical activity, as well as parental positive reinforcement for these behaviors. For a full description of the randomized controlled trial, see ref. (2).

The institutional review boards of San Diego State University and Southern California Kaiser Permanente (a referral source) approved this study. Participating parents and children provided written informed consent and assent, respectively.

\section{Measures}

Weight outcomes: At baseline, post-FBT, post-MT, and 2-year follow-up ( 2 years following the end of FBT), children's weight and height were measured using a calibrated balance beam scale and a stadiometer. Child percent overweight (percent above the median BMI for age and sex) was computed (12).

Attendance: FBT and MT sessions attended were tracked and summed, with in-person make-up sessions in the same week considered "attending."

Adherence: At post-FBT and post-MT, children and parents each completed a brief age-appropriate treatment-specific adherence questionnaire designed for the study (available upon request). Participants reported how often they had engaged in treatment-prescribed behaviors in the previous month (post-FBT) or two months (postMT), from never to always (0-4). MT subscales were derived by grouping items designed to assess each type of targeted behavior in BSM (self-regulatory/goal-setting, and problem-solving/cognitive- restructuring/relapse-prevention) and SFM (social support and body esteem/coping with teasing). The BSM parent measure also included a parenting skills subscale. Each MT subscale contained 4-11 items and demonstrated adequate internal consistency $(\alpha=0.59-0.85)$.

\section{Statistical analyses}

Analyses were performed using IBM SPSS v19.0. ANOVA and $t$-tests compared overall BSM vs. SFM adherence and subscales within each MT group. Separate hierarchical regression models were run for attendance and adherence, each predicting change in child percent overweight, at post-treatment and 2 years post-FBT. See Table 1 for variables examined, which included (i) total attendance; (ii) FBT adherence; (iii) child BSM, $n=41$, or SFM, $n=42$, individual adherence subscales; and (iv) parent BSM, $n=43$, or SFM, $n=42$, individual adherence subscales. Separate BSM and SFM models were needed, as MT adherence questionnaires were treatment-specific. Of the 101 MT families, child percent overweight data were available from 86 families at 2-year post-FBT. Intent-totreat analyses were also performed, using the last observation carried forward (e.g., child percent overweight at post-MT was used to replace missing values at 2-year follow-up), which enabled use of the full sample $(N=101)$. Secondary analyses examined the impact of including child sex and age in statistically significant regression models.

\section{Results \\ Attendance}

Participants attended a median of 17/20 FBT sessions (85\%) and 12/ 16 MT sessions (75\%). The median number of BSM and SFM sessions attended did not significantly differ $(75 \%$ vs. $69 \%$, respectively; $P=0.19$ ); thus, participants were pooled for MT attendance analyses and MT condition (BSM vs. SFM) was included as step 1 of the model. Higher total (FBT and MT) attendance was associated with a greater decrease in child percent overweight from baseline to post-MT, $\beta=-0.34, t(95)=-3.46, P=0.001$, although not from baseline to 2-year follow-up, $\beta=-0.08, t(83)=-0.74, P=0.461$. Results were identical when using intent-to-treat analyses $(N=$ 101), from baseline to post-MT $(P<0.001)$ and baseline to 2 -year follow-up $(P=0.380)$. Adding child sex to the model predicting percent overweight change from baseline to post-FBT did not alter this finding, although boys demonstrated greater percent overweight decreases, $\beta=0.23, t(94)=2.44, P=0.016$.

\section{Adherence}

The most common FBT skills reported by children were being physically active with friends, controlling portion sizes, limiting intake of high-fat/high-calorie foods, and self-monitoring. Parents most frequently reported praising children for healthy diet/activity behaviors, providing healthy foods for the family, and self-weighing. Both child and parent FBT adherence predicted greater child percent overweight decreases from baseline to post-FBT, although not from baseline to 2-year follow-up (Table 1). Adding child sex did not significantly impact the model, but boys had better post-FBT percent overweight decreases, $\beta=0.26, t(88)=2.59, P=0.011$.

In BSM, children, $t(40)=5.18, P<0.001$, and parents, $t(42)=$ 2.73, $P<0.01$, reported engaging in self-regulatory/goal-setting behaviors (e.g., self-monitoring, self-weighing, "getting back on track" if above weight maintenance range) more often than 
TABLE 1 Adherence during FBT and MT as predictors of child short- and long-term change in percent overweight

\begin{tabular}{|c|c|c|c|c|c|c|c|c|c|c|c|c|}
\hline \multirow[b]{2}{*}{ Predictor } & \multicolumn{12}{|c|}{ Time point } \\
\hline & $B$ & SEB & $\beta$ & $\Delta \mathrm{R}^{2}$ & $B$ & SEB & $B$ & $\Delta \mathrm{R}^{2}$ & $B$ & SEB & $\beta$ & $\Delta \mathrm{R}^{2}$ \\
\hline Step 1: Child FBT adherence & -3.16 & 1.21 & -0.26 & $0.06^{*}$ & - & & & & -2.17 & 2.64 & -0.09 & 0.01 \\
\hline Step 2: Parent FBT adherence & -3.11 & 1.56 & -0.23 & $0.08^{\star}$ & - & & & & -4.09 & 3.47 & -0.16 & 0.02 \\
\hline \multicolumn{13}{|l|}{ MT adherence: BSM } \\
\hline Child self-regulatory/goal-setting & - & & & & -0.22 & 0.85 & -0.04 & $<0.01$ & -9.32 & 3.35 & -0.44 & $0.19^{\star \star}$ \\
\hline $\begin{array}{l}\text { Child problem-solving/cognitive- } \\
\text { restructuring/relapse-prevention }\end{array}$ & - & & & & -0.04 & 0.74 & -0.01 & $<0.01$ & -1.03 & 0.85 & -0.19 & $<0.01$ \\
\hline Parent self-regulatory/goal-setting & - & & & & -1.03 & 0.85 & -0.19 & 0.04 & -3.19 & 3.44 & -0.15 & 0.02 \\
\hline $\begin{array}{l}\text { Parent problem-solving/cognitive- } \\
\text { restructuring/relapse-prevention }\end{array}$ & - & & & & -0.92 & 0.94 & -0.15 & 0.02 & -3.18 & 3.87 & -0.13 & 0.02 \\
\hline \multicolumn{13}{|l|}{ MT adherence: SFM } \\
\hline Child social support & - & & & & 0.52 & 0.99 & 0.08 & 0.01 & 1.48 & 2.73 & 0.09 & 0.01 \\
\hline $\begin{array}{l}\text { Child body esteem/coping } \\
\text { with teasing }\end{array}$ & - & & & & 0.08 & 0.87 & 0.02 & $<0.01$ & 2.92 & 2.33 & 0.21 & 0.04 \\
\hline Parent social support & - & & & & -2.62 & 1.49 & -0.27 & 0.07 & -0.20 & 1.87 & -0.02 & $<0.01$ \\
\hline $\begin{array}{l}\text { Parent body esteem/coping } \\
\text { with teasing }\end{array}$ & - & & & & -0.20 & 1.87 & -0.02 & $<0.01$ & 2.13 & 5.43 & 0.07 & $<0.01$ \\
\hline
\end{tabular}

$B$ and SEB are the $\beta$ coefficients, and $\beta$ is the standardized $\beta$ coefficient. The increase in the proportion of variance accounted for in the model by each predictor variable is indicated by $\Delta \mathrm{R}^{2}$. A negative $\beta$ weight indicates that higher adherence was associated with better child weight outcomes (i.e., a greater decrease or less of an increase in child percent overweight). Results were identical when using intent-to-treat analyses with last observation carried forward for post-MT and 2-year follow-up findings (all 101 families already had complete data at post-FBT).

BSM, behavioral skills maintenance treatment; FBT, family-based behavioral weight loss treatment; MT, maintenance treatment; SEB, standard error of B; SFM, social facilitation maintenance treatment.

${ }^{\star} P<0.05 .{ }^{*} P<0.01$

problem-solving/cognitive-restructuring/relapse-prevention behaviors (e.g., changing negative thoughts about eating/activity, brainstorming solutions to problems). BSM parents reported using parenting skills behaviors (e.g., praising children for making healthy choices) more often than self-regulatory/goal-setting behaviors, $t(42)=-2.04, P<$ 0.05 , or problem-solving/cognitive-restructuring/relapse-prevention behaviors, $t(42)=-4.75, P<0.001$. Only higher adherence to selfregulatory/goal-setting skills among BSM children predicted a decrease in child percent overweight from baseline to 2-year followup, $\beta=-0.44, t(33)=-2.78, P<0.01$ (Table 1), including within intent-to-treat analyses, $\beta=-0.38, t(39)=-2.57, P=0.014$. Child sex $(P=0.456)$ was nonsignificant when added to this model. Given that younger children may be less able to independently engage in self-regulatory strategies examined, this model was rerun among BSM children under age 10 years with adherence data $(n=$ 19) and the same pattern was observed, $\beta=-0.51, t(17)=-2.46$, $P<0.05$.

In SFM, children and parents reported similar usage of social support (e.g., making healthier choices when with friends/family, asking for support) and body esteem/coping with teasing skills (e.g., practicing ways to handle teasing, trying physical activities that were previously avoided), $P$ s $>0.11$. Neither child nor parent
SFM adherence subscales predicted child short- or long-term outcome.

\section{Discussion}

Attendance and self-reported BS adherence were related to children's short- and long-term weight outcomes, respectively, within an extended family-based weight control treatment. This extended weight control treatment previously has been shown to produce sustained improvements in children's relative weight as well as a range of psychosocial variables (2); the present study builds upon these findings to show that higher post-MT child-reported self-regulatory/ goal-setting skills usage predicted long-term but not post-treatment outcome. During treatment, regular contact with treatment providers (i.e., session attendance) may maximize children's weight control, whereas high adherence to BS targeted by the program differentiated children who were most successful after treatment contact ended, perhaps via skills mastery throughout contexts outside the clinic (13). Children's adherence did not significantly predict outcomes in SFM, which may reflect the distinct BSM- and SFM-specific adherence measures. SFM produces sustained weight control and domainspecific improvements in socially focused outcomes, including 
validated measures of peer support for healthy behaviors and teasing-related problem-solving (2). However, the present SFM adherence assessment-including behaviors that may appropriately change over time as social support improves-may not have sufficiently captured families' actual level of relevant adherence. Children may more easily recall engaging in self-regulatory and goal-setting skills (e.g., dietary monitoring and self-weighing) due to greater familiarity with FBT techniques that were continued throughout BSM. As self-regulatory skills predicted children's long-term outcome, integrating these skills into a comprehensive socially focused weight control program may produce the most robust weight outcomes $(14,15)$.

Including child age and sex did not appear to impact findings, although it is likely that the present analyses were underpowered to detect age differences within the age group examined (7-12 years, $N$ $=101)$. Children of this age require varying degrees of parental involvement and instruction when attempting self-regulatory strategies (e.g., self-monitoring of dietary intake or weight). Findings support the utility of emphasizing these crucial skills within familybased weight control interventions, including capitalizing on parental modeling and guidance, especially for younger children. Considering the importance of these skills for sustained weight control, it likely would provide added benefit to integrate self-regulatory strategies throughout treatment (e.g., by continually monitoring families' adherence to self-regulatory/goal-setting behaviors to reinforce their use and problem-solve around potential barriers to adherence. Although a higher proportion of children in the MT were girls, male children achieved even better weight outcomes; replication of longitudinal adherence analyses within larger samples would clarify whether findings equally apply to boys and girls.

Higher attendance predicted better child weight outcomes at posttreatment, but - in contrast to a study by Kalarchian and colleagues (3) - not at long-term follow-up. Of note, however, the present sample's attendance rate was higher and therefore more restricted in range (e.g., FBT dropouts, who had poorer FBT attendance, were not randomized to MT). Attendance is likely only a proxy for treatment adherence, which has been measured in a myriad of ways, including self-reported adherence to treatment components, as in the present study, and self-monitoring log completion or home food environment assessments $(8,10)$.

Adherence measures were relatively brief and treatment-specific (i.e., families in BSM and SFM completed distinct questionnaires), and only assessed behaviors in the past month (for FBT) or two months (for MT). Further, adherence questionnaires relied on retrospective self-report (or parent-report on their children), whereas other methods (e.g., observations collected via home assessment or real-time monitoring via telephone) might have enhanced validity and lowered measurement error. Finally, some SFM children likely successfully received social support or experienced less teasing by the end of treatment, which then might have led them to report less usage of some socially based skills (e.g., asking for support, practicing ways to handle teasing) at post-MT (2). Future studies should examine adherence as a mediator of outcome, using multi- ple assessments throughout treatment rather than solely at posttreatment.

Further studies are needed to identify the most potent strategies for improving families' adherence. Mastery-based education or programs of increased dose and/or duration may enhance adherence by allowing sufficient practice and integration of newly learned skills into families' daily habits. An extended, comprehensive maintenance program combining both behaviorally and socially based treatment targets is likely to maximize children's long-term weight control success (14). 0

\section{Acknowledgments}

This work was supported by NIH grants R01HD036904 (NICHD) and K24MH070446 (NIMH) to D.E.W.; K.R.T. and A.B.G. were supported by NIH grant T32HL007456 (NHLBI), and R.I.S. was supported by NIH grants KL2RR024994 and UL1RR024992. Portions of this manuscript were presented at the annual meeting of the Eating Disorders Research Society in Pittsburgh, PA (October 2007).

(c) 2012 The Obesity Society

\section{References}

1. Ogden CL, Carroll MD, Curtin LR et al. Prevalence of overweight and obesity in the United States, 1999-2004. JAMA 2006;295:1549-1555.

2. Wilfley DE, Stein RI, Saelens BE et al. Efficacy of maintenance treatment approaches for childhood overweight: a randomized controlled trial. J Am Med Assoc 2007;298(14):1661-1673.

3. Kalarchian MA, Levine MD, Arslanian SA et al. Family-based treatment of severe pediatric obesity: randomized, controlled trial. Pediatrics 2009;124:1060-1068.

4. Epstein LH, Wrotniak BH. Future directions for pediatric obesity treatment. Obesity (Silver Spring) 2010;18(Suppl 1):S8-12.

5. Martin KA, Bowen DJ, Dunbar-Jacob J, Perri MG. Who will adhere? Key issues in the study and prediction of adherence in randomized controlled trials. Control Clin Trials 2000;21:195S-199S.

6. Golan M, Kaufman V, Shahar DR. Childhood obesity treatment: targeting parents exclusively v. parents and children. Br J Nutr 2006;95:1008-1015.

7. Wrotniak BH, Epstein LH, Paluch RA, Roemmich JN. The relationship between parent and child self-reported adherence and weight loss. Obes Res 2005;13: 1089-1096.

8. Saelens BE, McGrath AM. Self-monitoring adherence and adolescent weight control efficacy. Child Health Care 2003;32(2):137-152

9. Germann JN, Kirschenbaum DS, Rich BH. Child and parental self-monitoring as determinants of success in the treatment of morbid obesity in low-income minority children. J Pediatr Psychol 2007;32:111-121.

10. Steele MM, Steele RG, Hunter HL. Family adherence as a predictor of child outcome in an intervention for pediatric obesity: Different outcomes for self-report and objective measures. Child Health Care 2009;38:64-75.

11. Goldfield GS, Epstein LH. Management of obesity in children. In: Fairburn CG, Brownell KD (eds). Eating Disorders and Obesity: A Comprehensive Handbook, 2nd edn. Guilford: New York, NY, 2002, pp 573-577.

12. Kuczmarski RJ, Ogden CL, Grummer-Strawn LM et al. CDC growth charts: United States. Adv Data 2000;1-27.

13. Bouton ME. A learning theory perspective on lapse, relapse, and the maintenance of behavior change. Health Psychol 2000;19(Suppl 1): 57-63.

14. Wilfley DE, Van Buren DJ, Theim KR et al. The use of biosimulation in the design of a novel multilevel weight loss maintenance program for overweight children. Obesity (Silver Spring) 2010;18(Suppl 1):S91-S98.

15. Wilfley DE, Vannucci A, White EK. Early intervention of eating- and weightrelated problems. J Clin Psychol Med Settings 2010;17:285-300. 